\title{
Probing collective excitations in helium nanodroplets: Observation of phonon wings in the infrared spectrum of methane
}

\author{
S. Rudolph, G. Wollny, K. von Haeften, and M. Havenith ${ }^{\text {a) }}$ \\ Lehrstuhl für Physikalische Chemie II, Ruhr-Universität Bochum, Universitätsstrasse 150, \\ 44780 Bochum, Germany
}

(Received 11 October 2006; accepted 24 January 2007; published online 29 March 2007)

\begin{abstract}
The authors have recorded the $\nu_{3}$ infrared spectrum of methane in helium nanodroplets using our $\mathrm{cw}$ infrared optical parametric oscillator. In a previous paper, Nauta and Miller [Chem. Phys. Lett. 350, 225 (2001)] reported the observation of the monomer rovibrational transitions of methane in helium nanodroplets. Here, they report the observation of additional absorption bands in the frequency range between 2990 and $3070 \mathrm{~cm}^{-1}$ blueshifted compared to the monomer transitions. They attribute these absorption features to phonon wings of individual rovibrational transitions, i.e., the simultaneous excitation of collective excitation modes of the quantum fluid and the rovibrational excitation of the methane monomer in the helium nanodroplet. (C) 2007 American Institute of Physics. [DOI: 10.1063/1.2709887]
\end{abstract}

\section{INTRODUCTION}

The spectroscopy of molecules in helium nanodroplets is a well established research field. Helium nanodroplets provide a finite size laboratory to study the special effects of finite size quantum fluids. ${ }^{1,2}$ Next to the rapidly growing field of Bose-Einstein condensates helium nanodroplets serve as prototypes of finite quantum fluids. The spectroscopy of dopants in helium nanodroplets gives access to the observation of collective excitations in a finite size Bose fluid. ${ }^{3}$ The relevant theoretical concepts are described by Dalfovo and Stringari, ${ }^{1}$ and Brink and Stringari. ${ }^{4}$ The quantum nature of these systems becomes obvious at low temperature where the de Broglie wavelength exceeds the spacing between individual particles.

Helium nanodroplets have collective excitations, such as phonons (volume compressional modes), ripplons (surface modes), and vortices (rotation modes). The finite size of the helium nanodroplets leads to a discrete excitation spectrum. Due to their inherent symmetry collective excitations in helium nanodroplets can be classified according to the number of radial nodes $\left(n_{r}\right)$ and angular momentum quanta $(m, l) .{ }^{1}$ In this paper we will focus on excitations of phonons in helium nanodroplets. Phonons describe volume compression modes with the lowest energy being described by $n_{r}=1 \quad(m=l=0)$. The phonon and roton branch in liquid helium was predicted very early by Landau ${ }^{5}$ and later confirmed by inelastic neutron scattering experiments. ${ }^{6}$ Helium nanodroplets constitute a related prototype of finite size quantum fluids. ${ }^{1,2,7}$

The first experimental evidence of phonon excitation in the electronic excitation spectrum of $\mathrm{C}_{2} \mathrm{H}_{2} \mathrm{O}_{2}$ in helium nanodroplets was reported by Hartmann et al. ${ }^{8}$ The structure of the phonon wing was found to be consistent with the existence of a phonon-roton spectrum of a superfluid liquid droplet, which differs from that of a normal fluid. In the energy spectrum of a normal fluid, the energy and the wave

${ }^{a)}$ Electronic mail: martina.havenith@rub.de vector of the translational motion are related by a monotonic increase for low $k$ values. The energy spectrum of the superfluid helium is characterized by a maximum (maxon) and minimum (roton minimum). For helium nanodroplets a similar energy spectrum was found. The best agreement with the experimental data as obtained for $\mathrm{C}_{2} \mathrm{H}_{2} \mathrm{O}_{2}$ in helium nanodroplets was found assuming values of $E_{\text {roton }}=7.8 \mathrm{~K}$ and $E_{\text {maxon }}=15.1 \mathrm{~K}$ for the excitation energy of the roton and maxon in superfluid helium nanodroplets, respectively. ${ }^{8}$ This corresponds to a slight adjustment compared to bulk helium, where $E_{\text {roton }}=8.7 \mathrm{~K}$ and $E_{\text {maxon }}=13.7 \mathrm{~K}$ were found. In the electronic spectra the excitation of phonons is caused by the interaction of the dopant with the helium.

Using IR spectroscopy of doped molecules the excitation of surface excitations in helium nanodroplets has been studied. ${ }^{9,10}$ Recently, we reported a detailed experimental and theoretical study on the coupling between phonon excitations and the rotational motion of $\mathrm{CO}$ in helium nanodroplets. ${ }^{3}$ The coupling is shown to depend crucially on the strength and anisotropy of the molecule-helium interaction potential. ${ }^{3}$ The dopant molecule can be viewed as a rotating "handle" which excites He droplets by the coupling to the collective excitation modes of helium. This coupling leads to a line broadening of the vibrational-rotational transition of the molecule and a reduction of the effective rotational constant. In a further study of NO in helium nanodroplets it was shown that extremely sharp rovibrational transitions are found in the absence of any excess rotational excitation of the dopant molecule. ${ }^{11}$ Although it is expected that changes in the solute-solvent interaction potential upon vibrational excitation of the dopant molecule can also lead to excitation of phonons, they have not been reported so far.

Experimental evidence for coupling between rotational and phonon excitations has also been reported by Hoshina et al. ${ }^{12}$ In the IR spectrum of $\mathrm{CO}_{2}$ in helium nanodroplets an additional absorption band was found which was blueshifted by $14 \mathrm{~cm}^{-1}$ compared to the monomer transition. The band 
was assigned to a simultaneous excitation of the rovibrational excited state of the monomer and the quantized rotation of the surrounding helium solvation shell. The experimental observation was in agreement with the so-called toy model of Lehmann. ${ }^{13}$ This model describes coupling of the rotational motion of the monomer with the excitations of the helium within the first solvation shell. It anticipates a quantized in-plane rotation of a ring of $N$ helium atoms which are coupled to the rotational motion of the dopant. The excitation of the ring of helium atoms is restricted to integer multiples of $N \hbar$.

The infrared spectrum of methane in helium nanodroplets has been reported previously by Nauta and Miller. ${ }^{14}$ They measured the spectrum of the $\nu_{3}$-stretching vibration between 3000 and $3050 \mathrm{~cm}^{-1}$ using a $F$-center laser which yields a typical output power of $50 \mathrm{~mW}$ and reported the measurement of several rovibrational transitions of the methane monomer. Furthermore, they found additional absorption features which were attributed to higher methane aggregates, e.g., dimer or trimer.

The linewidth of the $\mathrm{CH}_{4}$ monomer transition is found to be considerably broadened (exceeding that of methane in parahydrogen by a factor of 4). ${ }^{14}$ In their paper Nauta and Miller proposed an efficient coupling of motions of methane to the elementary excitations of helium, which motivated our search for phonon wings in the infrared spectrum of methane.

We report here the measurement of phonon wings accompanying individual rovibrational transitions in the infrared spectrum of methane.

\section{EXPERIMENTAL RESULTS}

We have scanned the frequency range of the $\nu_{3}$ band of methane between 2990 and $3070 \mathrm{~cm}^{-1}$ using our infrared $\mathrm{cw}$ optical parametric oscillator (OPO) as a radiation source. The laser has been described in detail in a previous paper. ${ }^{15}$ The $\mathrm{cw}$ infrared OPO provides a maximum output power of $2 \mathrm{~W}$. This exceeds the typical output power of a $F$-center laser as was used in the previous study by at least a factor of 10-20. The pump laser is a commercial Nd:Yag master laser with a light wave amplifier, providing up to $14 \mathrm{~W}$ laser radiation at $1064 \mathrm{~nm}$. In a quasiphase matched LiNb crystal the pump wave is split into a signal and an idler wave. The laser can be scanned in two different operation modes.

The pump laser can be continuously tuned in a frequency of $1.5 \mathrm{~cm}^{-1}$. The signal wave is kept fixed which results in a continuous tuning of the idler wave over $1.5 \mathrm{~cm}^{-1}$. The frequency resolution is limited by the resolution of the pump laser which amounts to $\sim 1 \mathrm{MHz}$. For the present study it was essential to scan over a broad frequency range. Therefore, we used an alternative scanning method. For a given pump frequency the frequency of the idler wave depends on the temperature of the LiNb crystal, which can be varied between 150 and $250^{\circ} \mathrm{C}$ with an accuracy of $0.002{ }^{\circ} \mathrm{C}$. Typically the temperature was increased by $40{ }^{\circ} \mathrm{C}$ over a time of several hours. This "fast" scanning mode is accompanied by small mode jumps (of typically $0.1 \mathrm{~cm}^{-1}$ ). The entire tuning range amounts to $\sim 100 \mathrm{~cm}^{-1}$. The fre-

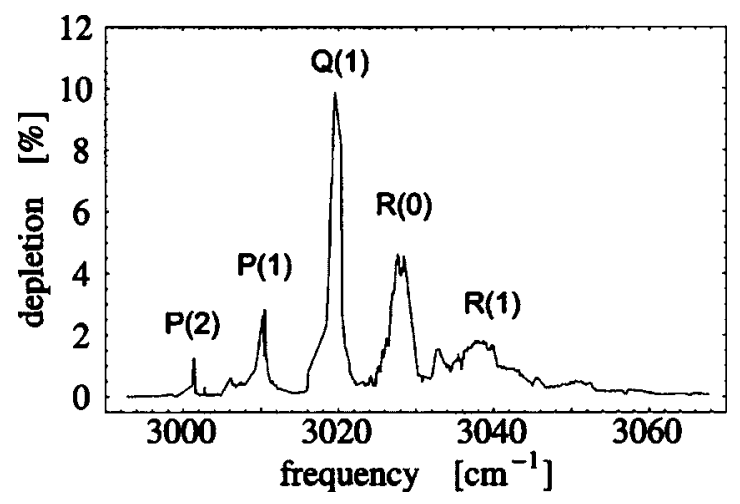

FIG. 1. IR spectrum of methane in helium nanodroplets with the assigned monomer transitions.

quency is measured by two Burleigh wavemeters which record simultaneously the wavelength of the pump and the signal wave.

The experimental setup of our helium nanodroplet machine has been described in detail in Ref. 3; therefore only a short overview will be given here. The helium nanodroplets were formed at a pressure of 40 bars and the nozzle $(5 \mu \mathrm{m}$ diameter) was kept at a temperature of $20 \mathrm{~K}$. This corresponds to a cluster with an average size of $\sim 2500 \mathrm{He}$ atoms. ${ }^{16}$ The IR laser radiation is focused into the helium cluster apparatus, such that the laser beam and the helium cluster beam are counter propagating. The laser was chopped with a frequency of $30 \mathrm{~Hz}$ and the total depletion signal was recorded with a mass spectrometer at mass 15 . We have installed a detector at the nozzle in order to measure the available laser power for depletion. Due to losses on the way from the laser to the skimmer in the helium cluster apparatus the maximum recorded laser power at this position in the interesting frequency range was $1 \mathrm{~W}$.

In Fig. 1 we display the experimental spectrum of methane in helium nanodroplets for increasing laser power (for the maximum laser power at a pickup pressure of 1.0 $\times 10^{-5} \mathrm{mbar}$ ). We have marked the monomer transitions at $3038.13,3029.07,3019.61$, and $3010.31 \mathrm{~cm}^{-1}$ which have been reported previously. ${ }^{14}$

At this higher laser power additional absorption features were observed at higher frequencies (e.g., at 3032 and above $3040 \mathrm{~cm}^{-1}$ ), which have not been seen in the previous study. These bands are increasing monotonically with increasing laser power (see Fig. 2). They are broad (up to several $\mathrm{cm}^{-1}$ ) and show no obvious substructure.

In order to test whether these bands can be attributed to IR transitions of clusters of methane or monomer lines we have systematically varied the doping pressure and measured the intensity of the IR transition. It is well known ${ }^{17,18}$ that the probability for the formation of smaller aggregates in the helium nanodroplets can in good approximation be described by the following Poisson distribution:

$$
P(n)=\frac{1}{n !}\left(\frac{\eta}{\eta_{1}}\right)^{n} \exp \left(-\frac{\eta}{\eta_{1}}\right)
$$

with $P(n), \eta_{1}, \eta$ being the probability to form a $\left(\mathrm{CH}_{4}\right)_{n}$ complex, $\eta_{1}$ the effective pressure necessary to pickup a single 


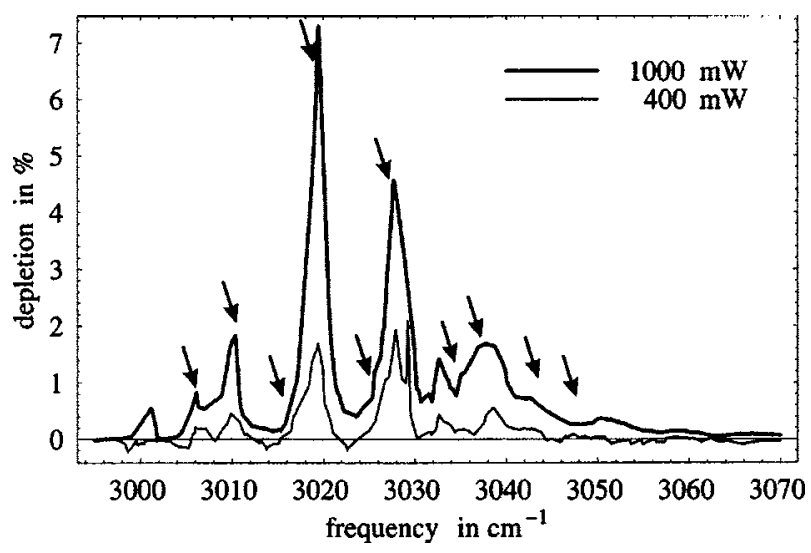

FIG. 2. IR spectrum of methane in helium nanodroplets in dependence of the laser power. Indicated are the predicted phonon wings. Some of them are overlapping with monomer transitions.

$\mathrm{CH}_{4}$ molecule, and $\eta$ the pickup pressure. As a consequence we expect at small pressures a linear increase of the intensity of monomer transitions with increasing pressure and a quadratic dependence for dimers.

Based on Eq. (1) we can carry out a more quantitative decomposition into the partial contribution from different cluster sizes, with less confidence for larger clusters. In this analysis, $\eta_{1}$ and the contributions of the different multimers were treated as fitting parameters. The latter were restricted to values between $n=1$ and $n=4$, since our main focus was to distinguish monomer contributions from contributions of multimers.

Nauta and Miller reported the observation of additional signals when increasing the pickup pressure. These lines were attributed to larger clusters $(n \geqslant 2)$ of methane. We have tested their assumption by measuring the change of signal intensity with increasing pressure for the transition at $3026 \mathrm{~cm}^{-1}$ (see Fig. 3). Our analysis yields a large contribution from the tetramer with a smaller contribution from the monomer, thereby confirming the previous assignment.

We now turn our attention to the new broad absorption bands, which are blueshifted compared to the monomer transitions around 3025,3035 , and $3045-3050 \mathrm{~cm}^{-1}$. Their intensity is increasing with increasing laser power. Since they are considerably weaker than the monomer transitions (factor of 10) they have not been observed before.

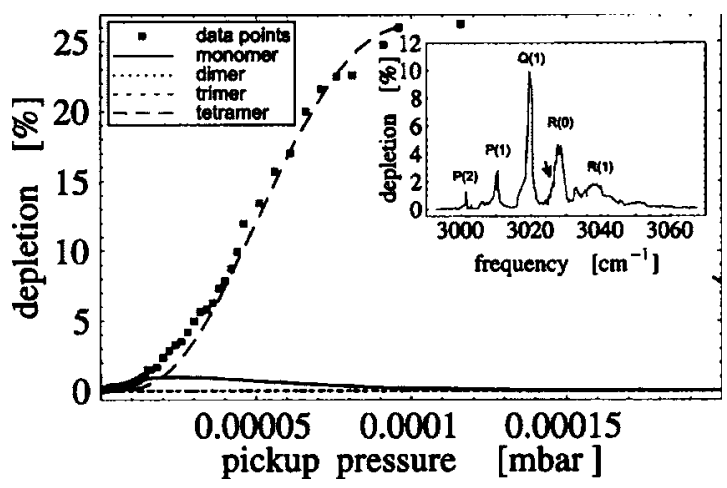

FIG. 3. Variation of the signal intensity in dependence of the methane pickup pressure at $3026.0 \mathrm{~cm}^{-1}$ (see arrow in inset). We see a strong contribution from the tetramer and a smaller contribution from the monomer.

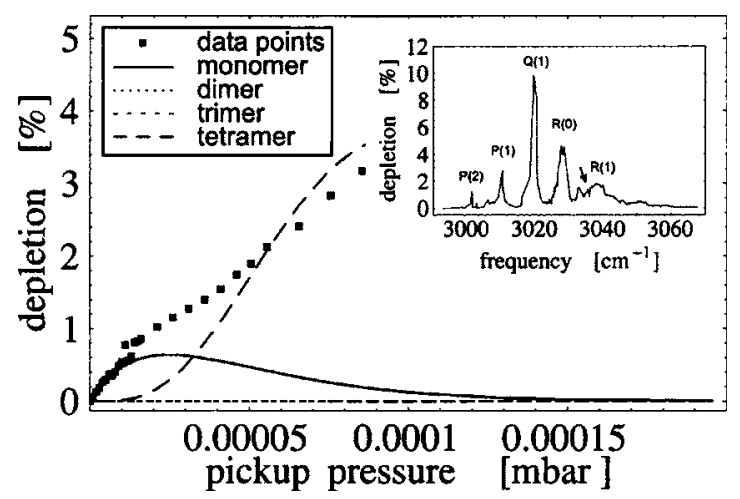

FIG. 4. The pickup pressure dependence of the broad absorption feature at $3035.3 \mathrm{~cm}^{-1}$ (see arrow in inset). This signal was attributed to the phonon wing of the $R(0)$.

First we have tested whether we can exclude impurities or methane-impurity complex formation as a possible source for the observed signals. We have added water and nitrogen which resulted in a decrease of the signal. Based on these results we can state that these transitions do not originate from water-methane or water-nitrogen complexes.

Furthermore, we have measured the change of the depletion signal with pickup pressure. In Figs. 4 and 5 the variations of the intensity with increasing methane pickup pressure are displayed at 3035.3 and $3044.2 \mathrm{~cm}^{-1}$, respectively. For low pressures we obtain a linear increase, which indicates the observation of monomer transitions. Based on this rigorous analysis we conclude that these bands have contributions from the monomer.

Due to its inherent tetrahedral symmetry methane has three different nuclear symmetries, $A, E$, and $F$, which should be populated according to their spin statistical weights, $5: 2: 3$, respectively. ${ }^{18}$ If we assume that only the lowest state for each symmetry ( $A$ symmetry, $J=0 ; F$ symmetry, $J=1$; and $E$ symmetry, $J=2)$ is populated at $0.37 \mathrm{~K}$, we expect to observe the $R(0), P(1), Q(1), R(1), P(2), Q(2)$, and $R(2)$ transitions of the monomer. Nauta and Miller reported the observation of the $R(1), R(0), Q(1)$, and $P(1)$ transitions. Their relative intensities are predicted to be 15 , 15,9 , and $3 .^{18}$ The predicted intensities for the $P(2), Q(2)$, and $R(2)$ are $2.8,2$, and 1.5 , respectively, which may explain why they have not been found previously.

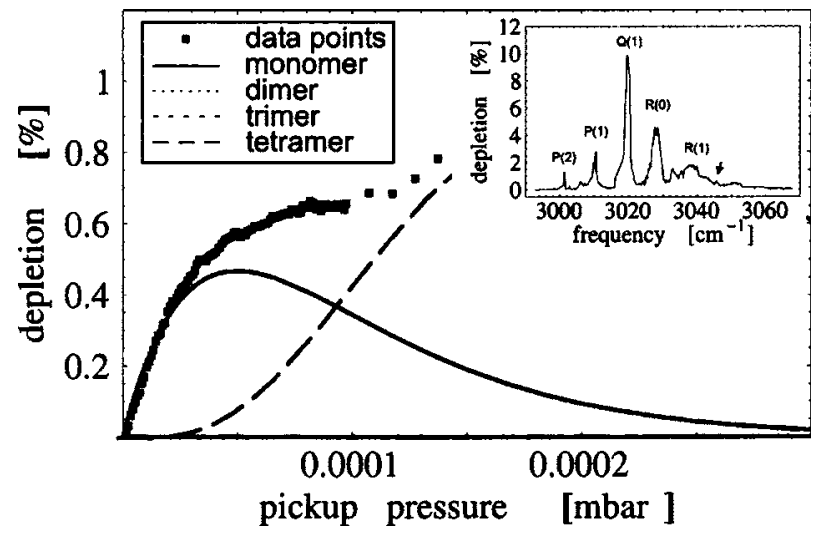

FIG. 5. The pickup pressure dependence of the broad absorption feature at $3044.2 \mathrm{~cm}^{-1}$ (see arrow in inset). This signal was attributed to the phonon wing of the $R(1)$. 
TABLE I. Rotational constants of methane in helium droplets.

\begin{tabular}{rrrr}
\hline \hline & Our results & $\begin{array}{r}\text { Results by Nauta } \\
\text { and Miller (Ref. 14) }\end{array}$ & $\begin{array}{r}\text { Gas phase } \\
\text { (Ref. 21) }\end{array}$ \\
\hline$\nu_{0}\left(\mathrm{~cm}^{-1}\right)$ & $3020.22(7)$ & $3020.32(2)$ & 3019.491 \\
$B^{\prime}\left(\mathrm{cm}^{-1}\right)$ & $4.998(38)$ & $4.932(10)$ & 5.196 \\
$B^{\prime \prime}\left(\mathrm{cm}^{-1}\right)$ & $4.986(38)$ & $5.012(10)$ & 5.241 \\
$D\left(\mathrm{~cm}^{-1}\right)$ & $0.014(5)$ & $0.0033(2)$ & 0.0001 \\
\hline \hline
\end{tabular}

If we take the rotational constants $B^{\prime}$ and $B^{\prime \prime}$ and centrifugal constant $D$ from the paper of Nauta and Miller, we predict the following transition frequencies for $P(2)$ and $R(2): \nu=3000.56 \mathrm{~cm}^{-1}$ and $\nu=3046.87 \mathrm{~cm}^{-1}$, respectively. A search for these transitions was carried out with $1 \mathrm{~W}$ laser power. In Fig. 2 the $P(2)$ transition can clearly be seen. The transition frequency is $3001.30 \mathrm{~cm}^{-1}$. We have included this new transition in a fit of the rotational constants using the same Hamiltonian as Nauta and Miller. ${ }^{18}$ The result of the fit is shown in Table I. Based on these constants we predict the $R(2)$ transition at $3046.53 \mathrm{~cm}^{-1}$. The experimental spectrum shows no obvious transition at this frequency. Only a weak, rather broad structure can be seen. This transition would be much broader than the $P(2)$ transition. However, the same tendency is found when comparing the line shape of the broad $R(1)$ transition with the much smaller $P(1)$ transition. One could speculate about possible relaxation channels in the upper rovibrational states; however, the experimental data are not obvious enough to allow further conclusions.

At higher laser power additional depletion signals are observed which are blueshifted from the monomer lines which cannot be attributed to additional monomer transition.

In the following discussion we will show that these additional bands can be attributed to rovibrational phonon wings.

\section{DISCUSSION}

We start with the toy model of Lehmann, ${ }^{13}$ which has been developed to describe the in-plane rotation of helium atoms around a linear molecule. A modification of this model for the correct treatment of a tetrahedral molecule would be beyond the scope of this paper. However, following the arguments of Lehmann we will discuss possible implications for the present example.

In the case of methane we consider a coupling of the methane rotation with a sphere of attached helium atoms. Following the calculations by Calderoni et al., ${ }^{19}$ we consider the rotation of a sphere with four helium atoms in agreement with the predicted minima in the $\mathrm{He}-\mathrm{CH}_{4}$ intermolecular potential energy surface. The minima correspond to the face configuration, with the $\mathrm{He}$ atom lying on the $C_{3}$ axis at a distance of $\left(r_{\min }\right)=6.44$ bohrs at the opposite side of the $\mathrm{He}$ atom. The rotational constant of methane in the gas phase is $B_{\mathrm{CH}_{4}}=5.26 \mathrm{~cm}^{-1}$. If we compute the rotational constants of a sphere with four He atoms fixed at the minimum configuration, we predict a rotational constant of $B_{\text {He-sphere }}$ $=0.135 \mathrm{~cm}^{-1}$.

Methane has a tetrahedral symmetry, which implies that we have to use the molecular symmetry group $T_{d}(M)$ to label the rotational vibrational levels. The rotational energy can be described by $E_{v}(j)=B j(j+1)$. Each level has a $(2 j+1)^{2}$ degeneracy. ${ }^{20}$ For methane the irreducible representation is given as $5 A 1 \oplus 5 A 2 \oplus 2 E \oplus 3 F 1 \oplus 3 F 2$ with the coefficients being the nuclear spin statistical weights. At room temperature, summed over all rotational states the population ratio is 5:2:9 for $A: E: F$, which will cool down to $j=0$ for $A$ symmetry, $j=1$ for $F$, and $j=2$ for $E$ symmetry. ${ }^{14}$

We can estimate the potential barrier for the rotation around its symmetry axis based on the intermolecular potential energy surface, as calculated by Calderoni et al. ${ }^{19}$ The minimum energy path for a rotation requires an energy that is equal to the difference between the energy minimum (face configuration: $-28.1 \mathrm{~cm}^{-1}$ ) and the transition state $\left(-21.5 \mathrm{~cm}^{-1}\right)$, i.e., $6.6 \mathrm{~cm}^{-1}$ per helium atom. If we account for four helium atoms the total required energy is $\Delta E$ $=26.4 \mathrm{~cm}^{-1}$. ${ }^{19}$

Lehmann has anticipated in his paper a potential of the form $V \cos \left(N\left(\theta_{1}-\theta_{2}\right)\right)$, with $V=\frac{1}{2} \Delta E$, e.g., $V=13.2 \mathrm{~cm}^{-1}$.

For a rotation of helium around the $C_{2}$ axis, the energy difference is predicted to be $E_{\text {face }}-E_{\text {vertex }}=13.7 \mathrm{~cm}^{-1}$ per helium atom. If we approximate this rotation by a potential of the form $V \cos (2(\theta))$, with $\theta$ describing the angle between the two $C_{2}$ axes, $V$ would be $\approx 13.7 \mathrm{~cm}^{-1}$, since two helium atoms contribute.

Lehmann describes in his paper two cases for which the energy can in good approximation be easily described. This two limiting cases are (a) the free rotor limit around the symmetry axes with a nearly free rotation of the helium sphere and (b) the strong coupling case with a strong coupling of the helium sphere rotation to the methane rotation.

The rotation of the methane monomer is described by the angular quantum number $j$ and the rotation of the helium sphere by the angular quantum number $l$. The total Hamiltonian is $H=B_{\mathrm{CH}_{4}} j(j+1)+B_{\mathrm{He}-\text { sphere }} l(l+1)+V$, with $B_{\mathrm{CH}_{4}}$ and $B_{\mathrm{He} \text {-sphere }}$ corresponding to the rotational constant of the methane atom and the helium sphere. The intermolecular potential $V$ leads to a coupling of both rotational angular momenta and hinders the free rotation of the methane.

Since the coupling strength $|V|=13.7 \mathrm{~cm}^{-1}$ is smaller than the internal energy $4\left(B_{\mathrm{CH}_{4}}+B_{\mathrm{He} \text {-sphere }}\right)=21.04 \mathrm{~cm}^{-1}$, we will in the following use the free rotor limit and obtain an energy of $B_{\mathrm{CH}_{4}} j(j+1)+B_{\mathrm{He} \text {-sphere }} l(l+1)$. In the free rotor limit the total wave function can be described by a product of two wave functions describing the rotation of the helium sphere and the methane molecules, separately.

For the case of a tetrahedral molecule $X Y_{4}$ with spin $I_{Y}$ $=0$, the spin statistical weights for the symmetries $A_{1}, A_{2}, E$, $T_{1}, T_{2}$ are 1:1:0:0:0, respectively. If we consider the rotation of four tetrahedrally arranged helium atoms around the $\mathrm{CH}_{4}$ molecule it is clear that the only allowed rotational levels correspond to $A_{1}$ and $A_{2}$ symmetries, e.g., $l=0\left(A_{1}\right), l$ $=3\left(A_{2}\right)$, and $l=4\left(A_{1}\right)$, with $l$ being the angular momentum quantum number of the internal rotation of the helium sphere. Rotational excitations with $l=1,2,5$ are not in agreement with the Bose symmetry of the He atoms. The rotational energies of the helium sphere for 
$l=0,3,4,6 \quad$ are $\quad E_{\mathrm{He}-\text { sphere }}(l=0)=0 \mathrm{~cm}^{-1}, \quad E_{\mathrm{He}-\text { sphere }}(l=3)$ $=1.61 \mathrm{~cm}^{-1}, E_{\text {He-sphere }}(l=4)=2.68 \mathrm{~cm}^{-1}$, and $E_{\text {He-sphere }}(l=6)$ $=5.63 \mathrm{~cm}^{-1}$, respectively.

For each $j$ value, a subset of levels with $l$ $=0,3,4,6,7, \ldots$, is expected. However, at $T=0.37 \mathrm{~K}$ only the lowest level for each symmetry (two levels) will be populated. The potential leads to a coupling of $j$ and $l$ to an overall angular momentum $J$. As a result each degenerate $j, l$ level will be split into several components with $J=\mid j$ $-l|, \ldots| j+l \mid$, , which are shifted in energy in dependence of the coupling strength.

For $j=0$, we obtain $J=0$; for $j=1$ we have $J=1 \quad(l=0)$, $J=2,3,4(l=3)$; and $J=3,4,5 \quad(l=4)$. The transition dipole moment is given by the vibrational excitation of the methane molecule. The helium is only indirectly coupled. The overall angular momentum $J$ can change by $\Delta J=0, \pm 1$. For a nearly free rotor strong transitions are expected for $\Delta l=0, j=1$, which correspond to the transitions, as reported by Nauta and Miller. ${ }^{14}$ The additional observed bands would have to be attributed to transitions with $\Delta l \neq 0$. The only allowed transitions from the ground state are $j=0, l=0, J=0 \rightarrow j=1, l$ $=0, J=1$. Further lines such as $j=0, l=3, J=3 \rightarrow j=1, l=3$, $J=2,3,4$ have almost the same transition frequency. For transitions originating in $j=1$, additional transitions can appear: $j=1, l=0, J=1 \rightarrow j=2, l=3, J=1,2$. These transitions are expected to be shifted by $\sim+1.61 \mathrm{~cm}^{-1}$. Transitions originating from $j=1, l=0, J=1 \rightarrow j=2, l=4, J=2$ are expected to be blueshifted by $2.68 \mathrm{~cm}^{-1}$. Additional transitions are not allowed since they would require $\Delta J \geqslant 1$.

Based on these considerations we could explain the presence of blueshifted "shoulders" for each monomer transition. However, we are unable to explain the broad features which are detected around 3035 and $3044 \mathrm{~cm}^{-1}$ within this model. In addition, rotation-phonon coupling is found to be quite unlikely based on the fact that the anisotropy in the potential is small compared to the cases where rotation-phonon coupling was found to be the dominating effect. ${ }^{3}$

Therefore, we will now follow a different approach to explain the results of our measurements.

In the electronic spectrum next to zero phonon lines Hartmann $e t a l .{ }^{8}$ observed phonon wings blueshifted by 6.2 and $9.2 \mathrm{~cm}^{-1}$. A combination band of $\mathrm{CH}_{4}$ vibrational excitation and phonon excitation would be a second possible explanation model.

If we anticipate a weak coupling for each rovibrational transition simultaneous excitation of rovibrational and phonon excitation is expected to be blueshifted by approximately equal to similar frequency shifts of 6.2 and $9.2 \mathrm{~cm}^{-1}$. These energies correspond to the energies where the density of states for the elementary excitations reaches its maximum. The predicted transition frequencies for a simultaneous excitation of vibration rotation of $\mathrm{CH}_{4}$ and phonons are listed in Table I and indicated in Fig. 2.

We can now check this assumption by measuring the changes in the depletion signal with increasing pickup pressure at these frequencies. At 3018.6, 3029.2, and $3038.8 \mathrm{~cm}^{-1}$ we find a very strong contribution from the monomer. However, these predicted bands overlap with the rovibrational monomer transitions. At the additional pre-
TABLE II. Listed are the monomer frequencies along with their predicted accompanying phonon wing. The transitions with a star have been observed previously (Ref. 14).

\begin{tabular}{lccc}
\hline \hline & $\mathrm{CH}_{4}$ transition & $+6.2 \mathrm{~cm}^{-1}$ & $+9.6 \mathrm{~cm}^{-1}$ \\
\hline$P(2)\left(\mathrm{cm}^{-1}\right)$ & 3001.3 & 3007.5 & 3010.9 \\
$P(1)\left(\mathrm{cm}^{-1}\right)$ & 3010.31 & 3016.5 & 3019.9 \\
$Q(1)\left(\mathrm{cm}^{-1}\right)$ & 3019.61 & 3025.8 & 3029.2 \\
$R(0)\left(\mathrm{cm}^{-1}\right)$ & 3029.07 & 3035.3 & 3038.7 \\
$R(1)\left(\mathrm{cm}^{-1}\right)$ & 3038.13 & 3044.3 & 3047.7 \\
\hline \hline
\end{tabular}

dicted frequencies, at 3025.8, 3035.2, 3044.2, and $3047.7 \mathrm{~cm}^{-1}$, the measurements yielded a clear contribution from the monomer, as can be seen by the initial increase of the signal with increasing pressure (see Figs. 4 and 5). However, a closer inspection shows that the features are found in close proximity to the transitions reported in Table II. The measured intensities of these phonon wings in relative depletion amount to $0.5 \%-1 \%$.

The measured intensities of these phonon wings in relative depletion amount to $0.8 \%\left(3025.8 \mathrm{~cm}^{-1}\right), \quad 0.6 \%$ $\left(3035.2 \mathrm{~cm}^{-1}\right), \quad 0.3 \% \quad\left(3044.2 \mathrm{~cm}^{-1}\right), \quad$ and $0.4 \%$ $\left(3047.7 \mathrm{~cm}^{-1}\right)$. The relative intensities are in agreement with the calculated relative intensities of the corresponding monomer transitions $(15,15,9$, and 9$)$. This intensity ratio is further supporting the phonon wing model, which predicts individual phonon wings resembling the relative intensities of the corresponding monomer transitions and contradicts the toy model.

For the future it would be interesting to measure additional vibrational bands of $\mathrm{CH}_{4}$ and different isotopes. An observation of phonon wings with similar frequency shifts would further support our conclusions.

An unambiguous proof for the presence of rotational phonon wings would be the observation of IR-IR double resonance signals. The associated phonon wing should then give rise to a double resonance signal on the corresponding probe frequency and allow a better separation between the otherwise partially overlapping transitions. However, this would require a second IR-OPO laser which pumps the monomer transition.

In conclusion, we attribute the observed broad bands which are blueshifted from the monomer-rovibrational lines to phonon wings that are excited upon vibrational excitation. Their intensity is decreased by a factor of 10 compared to the rovibrational line of the monomer. The measurements show that, similar to electronic spectroscopy, phonon wings can be observed in infrared spectroscopy. This implies the existence of a coupling mechanism between the vibrational excitation of the dopant with phonon excitations in helium nanodroplets.

\section{ACKNOWLEDGMENTS}

The authors acknowledge helpful discussions on data analysis with Gerhard Schwaab. This work has been supported by the Deutsche Forschungsgemeinschaft through Project No. HA 2394/9-3 within SPP 1119. 
${ }^{1}$ F. Dalfovo and S. Stringari, J. Phys. Chem. B 115, 10078 (2001).

${ }^{2}$ Y. Kwon, P. Huang, M. V. Patel, D. Blume, and K. B. Whaley, J. Chem. Phys. 113, 6469 (2000)

${ }^{3}$ K. von Haeften, S. Rudolph, I. Simanovski, M. Havenith, R. Zillich, and B. Whaley, Phys. Rev. B 73, 54502 (2006).

${ }^{4}$ D. M. Brink and S. Stringari, Z. Phys. D: At., Mol. Clusters 15, 257 (1990).

${ }^{5}$ L. Landau, J. Phys. (USSR) 5, 185 (1941).

${ }^{6}$ R. A. Cowley and A. D. B. Woods, Can. J. Phys. 49, 177 (1971).

${ }^{7}$ E. Krotscheck and R. E. Zillich, J. Chem. Phys. 115, 10161 (2001).

${ }^{8}$ M. Hartmann, F. Mielke, J. P. Toennies, A. F. Vilesov, and G. Benedek, Phys. Rev. Lett. 76, 4560 (1996).

${ }^{9}$ M. Kunze, P. R. L. Marwick, N. Pörtner, J. Reuss, and M. Havenith, J. Chem. Phys. 116, 7473 (2002).

${ }^{10}$ C. Callegari, I. Reinhard, K. K. Lehman, G. Scoles, K. Nauta, and R. E. Miller, J. Chem. Phys. 113, 4636 (2000).

${ }^{11}$ K. von Haeften, A. Metzelthin, S. Rudolph, V. Staemmler, and M. Ha- venith, Phys. Rev. Lett. 95, 215301 (2005).

${ }^{12}$ H. Hoshina, J. Lucrezi, M. N. Slipchenko, K. E. Kuyanov, and A. F. Vilesov, Phys. Rev. Lett. 94, 195301 (2005).

${ }^{13}$ K. Lehmann, J. Chem. Phys. 114, 4643 (2001).

${ }^{14}$ K. Nauta and R. E. Miller, Chem. Phys. Lett. 350, 225 (2001).

${ }^{15}$ J.-S. Samson, G. Wollny, A. Bergner, A. Hecker, G. Schwaab, A. D. Wieck, E. Bründermann, and M. Havenith, Phys. Chem. Chem. Phys. 8, 753 (2006).

${ }^{16}$ J. Harms, J. P. Toennies, and F. Dalfovo, Phys. Rev. B 58, 3341 (1998).

${ }^{17}$ M. Lewerenz, B. Schilling, and J. P. Toennies, J. Chem. Phys. 102, 8191 (1995).

${ }^{18}$ K. Nauta and R. E. Miller, J. Chem. Phys. 113, 10158 (2000).

${ }^{19}$ G. Calderoni, F. Cargnoni, R. Martinazzo, and M. Raimondi, J. Chem. Phys. 121, 8261 (2004).

${ }^{20}$ J. M. Hollas, High Resolution Spectroscopy (Wiley, West Sussex, 1998).

${ }^{21}$ W. L. Barnes, J. Susskind, R. H. Hunt, and E. K. Plyler, J. Chem. Phys. 56, 5160 (1972). 